\title{
Production and azimuthal anisotropy of muons from heavy flavor decays in small and large systems with ATLAS
}

\author{
Qipeng Hu, on behalf of the ATLAS Collaboration ${ }^{a, b, *}$ \\ ${ }^{a}$ University of Colorado Boulder, \\ 2000 Colorado Ave, Boulder, US \\ ${ }^{b}$ Lawrence Livermore National Laboratory, \\ 7000 East Ave, Livermore, US \\ E-mail: qipeng.hu@cern.ch
}

Heavy flavor production and collectivity in relativistic heavy-ion collisions collisions provide insight into the energy loss mechanism and transport properties of heavy quarks in the QGP medium. In these proceedings, ATLAS measurements on the elliptic flow coefficient, $v_{2}$, of muons from charm- and bottom-hadron decays in $\mathrm{Pb}+\mathrm{Pb}$ and $p p$ collisions are presented. Muons with charm and bottom origins are separated based on the transverse impact parameter with respect to the primary collision vertex. The event-plane method is used to extract flow coefficients in $\mathrm{Pb}+\mathrm{Pb}$ collisions, while a template fit method is used in $p p$ collisions to subtract non-flow contributions using a simultaneous fit to low and high charged-particle multiplicity samples. The extracted $v_{2}$ coefficients are presented as a function of muon $p_{\mathrm{T}}$ and event activity.

HardProbes 2020

1-6 June 2020

Austin, Texas

${ }^{*}$ Speaker 


\section{Introduction}

The quark-gluon plasma (QGP) is a state of matter in which the quarks and gluons are deconfined from color neutral hadronic states. Relativistic heavy-ion collisions create nuclei-sized droplets of QGP. In these collisions, heavy quarks, charm and bottom, are mostly produced via high-momentumtransfer interactions between incident initial quarks and gluons in nuclei due to their large masses. Once created, these quarks will experience substantial modifications to their transverse momentum $\left(p_{\mathrm{T}}\right)$ and azimuthal angle $(\phi)$ distributions when interacting with the QGP through both radiative and collisional processes, but they cannot be destroyed. The surviving heavy quarks provide information on the strength of the interactions and constituents of the QGP. In measurements of the production of prompt $D^{0}$ mesons in lead-lead $(\mathrm{Pb}+\mathrm{Pb})$ collisions at the Large Hadron Collider (LHC), large $p_{\mathrm{T}}$ modification and azimuthal anisotropy, quantified by the nuclear modification factor $\left(R_{\mathrm{AA}}\right)$ and flow coefficients $\left(v_{n}\right)$, have been observed [1-4]. A number of models considering a strong interaction of heavy quarks inside the QGP medium can roughly describe the measured $R_{\mathrm{AA}}$ and second flow coefficient $v_{2}$ as discussed in recent reviews [5]. Due to the small size of proton-lead collisions, only a small droplet or no QGP is expected to form. Similar studies of heavy flavor production in proton-lead collisions at the LHC show little modification of the $p_{\mathrm{T}}$ distribution but sizable azimuthal anisotropy [6,7], which cannot be described by the same theoretical frameworks used to describe $\mathrm{Pb}+\mathrm{Pb}$ collisions. To better understand the origin of the azimuthal anisotropy of heavy flavors in nucleon-sized small collision systems, $v_{2}$ for muons from heavy flavor decays is measured in the even smaller collision system of proton-proton $(p p)$. In these proceedings, ATLAS measurements of $v_{2}$ of muons from charm- and bottom-hadron decays in $\mathrm{Pb}+\mathrm{Pb}$ collisions [8] and high multiplicity $p p$ collisions [9] are reported.

\section{Analysis}

The ATLAS experiment consists of an inner tracking detector (ID) surrounded by a thin superconducting solenoid, electromagnetic and hadron calorimeters, and a muon spectrometer (MS). The MS includes a system of precision tracking chambers and fast detectors for triggering. The first-level trigger is implemented in hardware and uses a subset of the detector information. This is followed by the software-based high level trigger.

Muons are selected from events triggered by online muon-like object in the MS. Successful ID and MS muon track combination and tight quality cuts are further required to suppress fake muons. The residual background contribution, primarily from hadronic punch-through, is separated statistically using the momentum imbalance, $\Delta p / p_{\mathrm{ID}}=\left(p_{\mathrm{ID}}-p_{\mathrm{MS}}\right) / p_{\mathrm{ID}}$, where $p_{\mathrm{ID}}$ is the momentum measured in the ID, and $p_{\mathrm{MS}}$ is that measured in the MS and corrected for the energy loss inside the calorimeter. Muons from heavy-flavor hadron decays can be further separated into charm- and bottom-hadron decays based on the displacement of the muon track from the collision vertex, which is quantified by the transverse impact parameter, $d_{0}$. Momentum imbalance and transverse impact parameter distributions of muons from charm- and bottom-hadron decays and from background are predicted by PYтнIA 8 generated events with full ATLAS detector response simulation.

In the analysis of the $\mathrm{Pb}+\mathrm{Pb}$ data at $\sqrt{s_{\mathrm{NN}}}=5.02 \mathrm{TeV}$ collected in 2015 and 2018, the so-called event-plane method is used to extract flow coefficients. The event-plane angle, $\Psi_{n}$ at $n^{\text {th }}$ order, is 
measured using the angular distribution of the energy deposited in the forward calorimeter (FCal) towers, covering pseudorapidity $(\eta)$ of $3.1<|\eta|<4$.9. Yields of muons from charm- and bottomhadron decays are extracted in bins of relative angle between the muon and event-plane angle $\left(\phi-\Psi_{n}\right)$. Modulations from a uniform relative angle distribution represent the anisotropy of muon production over the entire event. The Fourier coefficients decomposed from the angular modulation are further corrected by the event-plane resolution. The event-plane resolution is determined by the difference in event-plane angles in each individual FCal system on the two sides of the ATLAS detector. The resolution corrected Fourier coefficients are taken as the measured flow coefficients.

In the analysis of the $2017 p p$ data at $\sqrt{s}=13 \mathrm{TeV}$, where the event-plane angle cannot be determined reliably, the two-particle correlation methods are utilized. Selected muons including background are correlated with ID tracks in relative azimuthal angle $(\Delta \phi)$ and relative pseudorapidity $(\Delta \eta)$ to form the correlation function. Correlations from particle pairs in jets and resonance decays are largely suppressed by a pseudorapidity gap of $|\Delta \eta|>1.5$. The residual non-flow contribution is dominated by particle pairs from back-to-back jets, and is subtracted using a template fit method to a one-dimensional correlation function in $\Delta \phi$. The shape of the correlation function from backto-back jets is assumed to be unchanged with event multiplicity $\left(N_{\mathrm{ch}}^{\mathrm{rec}}\right)$, where the multiplicity is defined as the number of reconstructed ID tracks with $p_{\mathrm{T}}>0.4 \mathrm{GeV}$ associated with the vertex from which the muon originates. Thus, correlation functions at low multiplicity $\left(N_{\mathrm{ch}}^{\mathrm{rec}}<40\right)$ are used as a template of the non-flow contribution at high multiplicity and subtracted. Fourier coefficients in the residual correlation function are expected to be the same as those from the event-plane method. Background contribution removal and charm- and bottom-hadron origin separation are done statistically from the measured Fourier coefficients via extrapolation in various quantities which control the muon momentum imbalance and transverse impact parameter.

\section{Results}

The second order flow coefficients, $v_{2}$, of muons from charm- and bottom-hadron decays measured in $\mathrm{Pb}+\mathrm{Pb}$ collisions at $\sqrt{s_{\mathrm{NN}}}=5.02 \mathrm{TeV}$ are shown as a function of muon $p_{\mathrm{T}}$ in Figure 1. The left panel is for central collisions with centrality $0-10 \%$ and the right panel for semi-peripheral collision with centrality 40-60\%. Statistical uncertainties are shown as vertical lines and systematic uncertainties as boxes. The results indicate a non-zero $v_{2}$ for both the charm and bottom muons, with substantially larger elliptic flow coefficients for charm muons in both central and semi-peripheral $\mathrm{Pb}+\mathrm{Pb}$ collisions. The statistical and systematic uncertainties have a significant contribution that is anti-correlated between the charm and bottom $v_{2}$. The data in each centrality interval are compared with two sets of theoretical calculations: DREENA-в [10] and DAB-MOD [11]. The DREENA-B calculations include radiative and collisional energy loss of heavy quarks inside the medium and show reasonable agreement for the $v_{2}$ of muons from both charmand bottom-hadron decays. Calculations with the DAB-MOD framework consider only Langevin drag and diffusion contributions and underestimate the $v_{2}$ of muons from charm-hadron decays. The model qualitatively describes the similar $v_{2}$ between muons from charm- and bottom-hadron decays at high $p_{\mathrm{T}}$, but the $v_{2}$ value is about a factor of two lower than the DREENA-B results. Additional energy loss contributions to DAB-MOD, not included here, tend to increase the high- $p_{\mathrm{T}}$ anisotropies. 

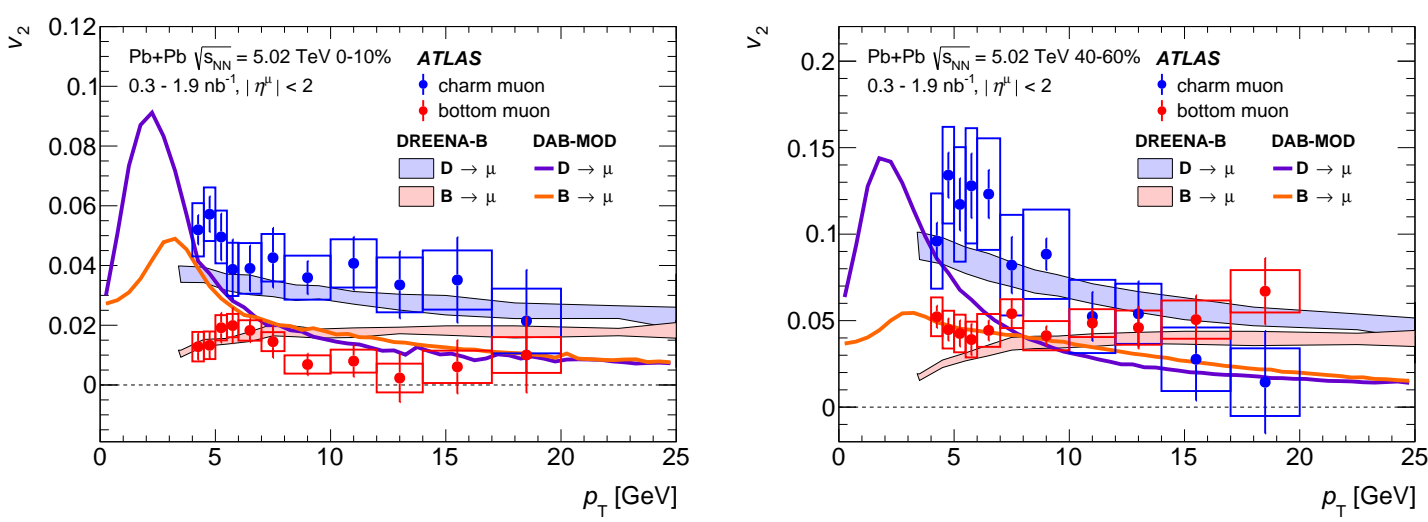

Figure 1: Elliptic anisotropy coefficient $v_{2}$ of muons from charm- and bottom-hadron decays as a function of $p_{\mathrm{T}}$ in the $5.02 \mathrm{TeV} \mathrm{Pb}+\mathrm{Pb}$ data for the $0-10 \%$ (left) and 40-60\% (right) centrality interval, compared with theoretical predictions based on DREENA-B [10] and DAB-MOD [11] in the same centrality intervals. This figure is from Ref. [8].

Figure 2 shows the measured $v_{2}$ of muons from charm- and bottom-hadron decays in high multiplicity $p p$ collisions $\sqrt{s}=13 \mathrm{TeV}$ as a function of muon $p_{\mathrm{T}}$ on the left panel and as a function of $N_{\mathrm{ch}}^{\text {rec }}$ on the right panel. Statistical uncertainties are shown as vertical lines and systematic uncertainties as shaded bands. The $v_{2}$ of muons from bottom-hadron decays is consistent with zero in the measured muon $p_{\mathrm{T}}$ range of $4<p_{\mathrm{T}}<7 \mathrm{GeV}$. However, the $v_{2}$ of muons from charm-hadron decays is found to be significantly non-zero at lower $p_{\mathrm{T}}$, decreasing with increasing $p_{\mathrm{T}}$ until it becomes consistent with zero at higher $p_{\mathrm{T}}$. The measured $v_{2}$ of muons from both charm- and bottom-hadron decays show little dependence on $N_{\mathrm{ch}}^{\mathrm{rec}}$ within the uncertainties. These results indicate that the origin of the anisotropy of heavy flavor quarks in $p p$ collisions has a strong dependence on the heavy quark mass.
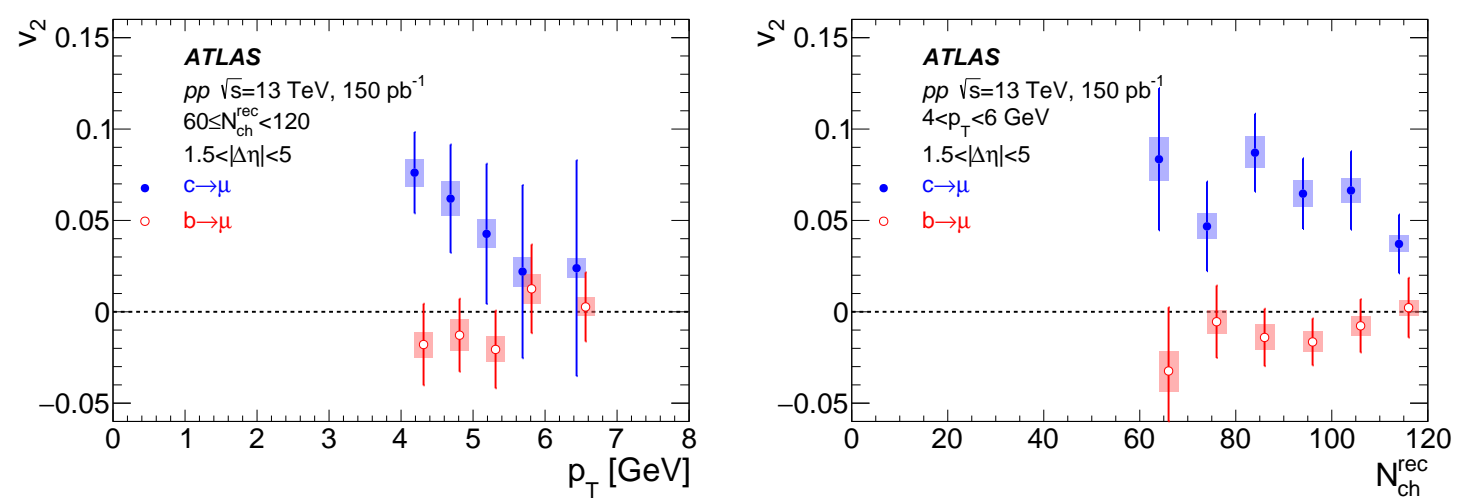

Figure 2: Elliptic anisotropy coefficient $v_{2}$ of muons from charm- and bottom-hadron decays as a function of $p_{\mathrm{T}}$ for the $60 \leq N_{\mathrm{ch}}^{\mathrm{rec}}<120$ multiplicity range (left) and as a function of track multiplicity $N_{\mathrm{ch}}^{\mathrm{rec}}$ for muons with transverse momentum $4<p_{\mathrm{T}}<6 \mathrm{GeV}$ (right). Data points are shifted by \pm 1 in $N_{\mathrm{ch}}^{\mathrm{rec}}$ and $\pm 0.125 \mathrm{GeV}$ in $p_{\mathrm{T}}$ for better visibility. The vertical bars and shaded bands represent statistical and systematic uncertainties, respectively. This figure is from Ref. [9]. 


\section{Summary}

These proceedings summarize ATLAS measurements of the azimuthal anisotropy of muons from charm- and bottom-hadron decays in high multiplicity $p p$ collisions and $\mathrm{Pb}+\mathrm{Pb}$ collisions at the LHC. In $\mathrm{Pb}+\mathrm{Pb}$ collisions, a significant non-zero $v_{2}$ of muons from charm- and bottom-hadron decays is observed in both central and peripheral events. Muons from charm-hadron decays have larger $v_{2}$ than those from bottom-hadron decays at low $p_{\mathrm{T}}$. In high multiplicity $p p$ collisions, muons from charm-hadron decays show a significant $v_{2}$ which is independent of multiplicity and decreases with $p_{\mathrm{T}}$, while the $v_{2}$ of muons from bottom-hadron decays is consistent with zero within uncertainties.

\section{References}

[1] CMS Collaboration, Nuclear Modification Factor of $D^{0}$ Mesons in Pb-Pb Collisions at $\sqrt{s_{\mathrm{NN}}}=$ $5.02 \mathrm{TeV}$, Phys. Lett. B 782 (2018) 474 [1708.04962 [nucl-ex]].

[2] ALICE Collaboration, Measurement of $D^{0}, D^{+}, D^{*+}$ and $D_{s}^{+}$Production in $\mathrm{Pb}-\mathrm{Pb}$ Collisions at $\sqrt{s_{\mathrm{NN}}}=5.02 \mathrm{TeV}, \operatorname{JHEP} 10$ (2018) 174 [1804.09083[nucl-ex]].

[3] CMS Collaboration, Measurement of Prompt $D^{0}$ Meson Azimuthal Anisotropy in $\mathrm{Pb}-\mathrm{Pb}$ Collisions at $\sqrt{s_{\mathrm{NN}}}=5.02 \mathrm{TeV}$, Phys. Rev. Lett. 120 (2018) 202301 [1708.03497[nucl-ex]].

[4] ALICE Collaboration, D-meson Azimuthal Anisotropy in Mid-central Pb-Pb Collisions at $\sqrt{s_{\mathrm{NN}}}=5.02 \mathrm{TeV}$, Phys. Rev. Lett. 120 (2018) 102301 [1707.01005[nucl-ex]].

[5] X. Dong, Y.-J. Lee and R. Rapp, Open Heavy-Flavor Production in Heavy-Ion Collisions, Ann. Rev. Nucl. Part. Sci. 69 (2019) 417 [1903.07709 [nucl-ex]].

[6] CMS Collaboration, Elliptic Flow of Charm and Strange Hadrons in High-multiplicity p-Pb Collisions at $\sqrt{s_{\mathrm{NN}}}=8.16 \mathrm{TeV}$, Phys. Rev. Lett. 121 (2018) 082301 [1804.09767 [nucl-ex]].

[7] ALICE Collaboration, Measurement of Prompt $D^{0}, D^{+}, D^{*+}$, and $D_{s}^{+}$Production in $p-P b$ Collisions at $\sqrt{s_{\mathrm{NN}}}=5.02 \mathrm{TeV}$, JHEP 12 (2019) 092 [1906.03425 [nucl-ex]].

[8] ATLAS Collaboration, Measurement of Azimuthal Anisotropy of Muons from Charm and Bottom Hadrons in Pb+Pb Collisions at $\sqrt{s_{\mathrm{NN}}}=5.02 \mathrm{TeV}$ with the ATLAS Detector, Phys. Lett. B 807 (2020) 135595 [2003.03565[nucl-ex]].

[9] ATLAS Collaboration, Measurement of Azimuthal Anisotropy of Muons from Charm and Bottom Hadrons in p pollisions at $\sqrt{s}=13 \mathrm{TeV}$ with the ATLAS Detector, Phys. Rev. Lett. 124 (2020) 082301 [1909.01650 [nucl-ex]].

[10] D. Zigic et al., DREENA-B framework: First Predictions of $R_{A A}$ and $v_{2}$ within Dynamical Energy Loss Formalism in Evolving QCD Medium, Phys. Lett. B 791 (2019) 236 [1805.04786[nucl-ex]].

[11] R. Katz et al., Sensitivity Study of Heavy Flavor $R_{A A}$ and Azimuthal Anisotropies Based on Beam Energy, Initial Conditions, Hadronization, and Suppression Mechanisms, Phys. Rev. C 102 (2020) 024906 [1906 . 10768 [nucl-ex]]. 